Research Article

\title{
Fault Diagnosis of Oil Pumping Machine Retarder Based on Sound Texture-Vibration Entropy Characteristics and Gray Wolf Optimization-Support Vector Machine
}

\author{
Shutao Zhao, ${ }^{1}$ Ke Chang, ${ }^{1}$ Erxu Wang $\mathbb{D}^{D},{ }^{2} \mathrm{Bo} \mathrm{Li}^{1}{ }^{1}$ Kedeng Wang, ${ }^{1}$ and Qingquan $\mathrm{Wu}^{3}$ \\ ${ }^{1}$ School of Electrical and Electronic Engineering, North China Electric Power University, Baoding 071003, China \\ ${ }^{2}$ Baoding Power Supply Company of State Grid, Baoding, Hebei 071051, China \\ ${ }^{3}$ Rennan Oil Production Area, No. 1 Oil Production Plant, Huabei Oilfield Company, Cangzhou 062552, China
}

Correspondence should be addressed to Erxu Wang; 946197703@qq.com

Received 27 May 2019; Accepted 18 August 2019; Published 11 May 2020

Academic Editor: Davood Younesian

Copyright (c) 2020 Shutao Zhao et al. This is an open access article distributed under the Creative Commons Attribution License, which permits unrestricted use, distribution, and reproduction in any medium, provided the original work is properly cited.

In order to diagnose the retarder faults of oil pumping machine accurately in complex environments and improve the generalization of the algorithm, a GWO-SVM fault diagnosis algorithm based on the combination of sound texture and vibration entropy characteristics was proposed. Firstly, the acquired sound signal was purified by band-pass filter, then generalized S-transform was developed to extract the box dimension, directivity, and contrast ratio, which reflect the characteristics of time-frequency spectrum, to construct three-dimensional texture eigenvectors. Secondly, the $K$ parameter of variational mode decomposition (VMD) was reasonably selected by the energy method, and then the vibration signal was decomposed to get modal components, and the permutation entropy was obtained from modal components. Finally, joint eigenvectors were constructed and fed into SVM for learning. The gray wolf optimization (GWO) algorithm was used to optimize the parameters of the SVM model based on mixed kernel function, which reduces the impact of sensor frequency response, environmental noise, and load fluctuation disturbance on the accuracy of retarder fault diagnosis. The results showed that the GWO-SVM fault diagnosis method, which is based on the combination of sound texture and vibration entropy characteristics, makes full use of the complementary advantages of signal frequency band. And the overall diagnostic accuracy for the experimental samples reaches $100 \%$, which has good generalization ability.

\section{Introduction}

Oil pumping machine relies on the up and down movement of the horsehead to complete the lifting of the crude oil from the wellbore. The retarder connecting the crank train is the key component of the power transmission. Due to friction and impact, oil pumping machine is prone to oil leakage, gear damage, bearing damage, belt wear, and other faults $[1,2]$. Vibration signal characteristics can be used to diagnose mechanical failure of oil pumping machine retarder $[3,4]$. But limited to the charge accumulating effect and coupling mode of the piezoelectric accelerometer, it is likely to cause detection failure from the continuous impact caused by the pitting and broken cog of the retarder. The reason is that the vibration sensor only detects a narrow frequency response range and is insensitive to high-frequency signal changes. The sound signal accompanying the operation of the oil pumping machine is homologous to the vibration signal and can be obtained by the noncontact electret film capacitive sensor, which can effectively compensate for the detection failure phenomenon caused by the vibration sensor band limitation.

For nonstationary signals such as sound and vibration, there are mainly analysis methods such as dynamic time warping (DTW), wavelet transform (WT), empirical mode decomposition (EMD), and local mean decomposition (LMD) [5-8]. The DTW planning optimal path is prone to metamorphosis distortion, WT has energy leakage, and the two cannot adaptively decompose the signal. The EMD adaptive decomposition process is prone to over enveloping, end effect, and modal aliasing. LMD optimizes underfrequency, over envelopes, and other issues of EMD. 
However, LMD and EMD are essentially recursive decomposition methods, which cannot eliminate the endpoint effect, modal aliasing, and other issues fundamentally. Variational mode decomposition (VMD) is a new nonrecursive mode decomposition method, which avoids the modal aliasing problem caused by envelope error $[9,10]$. The generalized S-transform is developed by short-time Fourier transform (STFT) and Wigner-Ville distribution (WVD), which makes up for the defects of STFT single resolution and WT phase and without cross-term interference problem. It introduces a frequency-dependent adjustment factor into the window function to adjust the time-frequency resolution [11].

For the sound-vibration signal combination method, Zhao et al. [12] process sound and vibration signals via improving the ensemble empirical mode decomposition (EEMD) to calculate the two-dimensional spectral entropy. The diagnostic effect is improved. The literature [13] utilizes wavelet packet and feature entropy theory to extract the features of the collected sound and vibration signals, which improves the diagnostic accuracy. Zhang et al. [14] construct three-dimensional (3D) graph of sound and vibration and extract envelope of 3D graph and hierarchical eigenvectors based on shape, which provides a new idea for circuit breaker diagnosis. In [15], LMD is utilized to decompose sound and vibration signals and select appropriate PF component to obtain feature entropy as the eigenvectors. The diagnostic accuracy is improved. Although studies [12-15] have achieved some good results, the difference in sound and vibration signals is not considered. The feature extraction methods of the two signals are identical, which leads to misdiagnosis and poor generalization.

It is a beneficial attempt to distinguish the defect of oil pumping machine retarder by the features combination of sound and vibration signals. The generalized S-transform is very sensitive to high-frequency impact signals and has high time-frequency resolution [16], which can accurately reflect the high-frequency impact characteristics of sound signals and rich time-frequency information. The load fluctuation of oil pumping machine's up and down stroke causes the spectrum of the vibration signal of the retarder to be extremely complicated. The signal components include the combination of gear shaft rotation, gear meshing frequency, and so on [17]. Performing VMD method can effectively improve misdiagnosis and missed diagnosis caused by band aliasing. In this paper, the combined features of sound and vibration signals are extracted and combined with the GWO-SVM model for fault identification. The diagnosis process is shown in Figure 1.

AC144 piezoelectric acceleration sensor $(0.6-10000 \mathrm{~Hz})$ and NVL-AF-audio embedded waterproof (explosion-proof) high-fidelity pickup $(20-20000 \mathrm{~Hz})$ were acquired to collect CYJ10-3-48HB oil pumping machine retarder $(\mathrm{CJH} 1100 \times 73)$ multiple sets of sound and vibration sample signals under retarder oil leakage, gear pitting peeling, belt damage, and normal state to extract sound texture-vibration entropy characteristics, respectively.

\section{Sound Signal Feature Extraction}

The motor transmits power to the retarder through the belt. The retarder reduces the high-speed rotation of the motor through three-axis two-stage deceleration to the low-speed rotation of the crankshaft. The whole operation process is relatively complex, and it runs in the open air. As a result, the sound signals collected include the belt friction noise of oil pumping machine, the noise of the motor, wind noise, thunder noise, and human voice. Through spectrum analysis, it is not hard to find that the above noise is mostly low-frequency interference, and the frequency is concentrated between 0 and $10 \mathrm{kHz}$. So the frequency band noise of sound signals below $10 \mathrm{kHz}$ and above $20 \mathrm{kHz}$ is filtered by finite impulse response (FIR) band-pass filter. The sound signals in normal operation state between 10 and $20 \mathrm{kHz}$, before and after denoising, are shown in Figure 2.

2.1. Sound Signal Generalized S-Transform. The windowed Fourier transform for signal $x(t)$ is performed:

$$
S(f)=\int_{-\infty}^{+\infty} x(t) w(t) e^{-j 2 \pi f t} \mathrm{~d} t
$$

The Gauss window function is scaled $\sigma$ and translated $\tau$, that is,

$$
w(\tau-t)=\frac{1}{\sigma \sqrt{2 \pi}} e^{-\left((\tau-t)^{2} / 2 \sigma^{2}\right)} .
$$

The time-frequency spectrum of $x(t)$ is obtained by introducing formula (2) into formula (1):

$$
S(\sigma, \tau)=\int_{-\infty}^{+\infty} x(t) \frac{1}{\sigma \sqrt{2 \pi}} e^{-\left((\tau-t)^{2} / 2 \sigma^{2}\right)} e^{-j 2 \pi f t} \mathrm{~d} t .
$$

Set $\sigma(f)=1 /|f|$, get the S-transform as follows:

$$
\begin{aligned}
S(f, \tau) & =\int_{-\infty}^{+\infty} \frac{|f|}{\sqrt{2 \pi}} e^{\left(-f^{2}(\tau-t)^{2} / 2\right)} x(t) e^{-j 2 \pi f t} \mathrm{~d} t \\
& =\int_{-\infty}^{+\infty} \omega(f,(\tau-t)) x(t) e^{-j 2 \pi f t} \mathrm{~d} t, \\
\omega(f, \tau-t) & =\frac{|f|}{\sqrt{2 \pi}} e^{\left(-f^{2}(\tau-t)^{2} / 2\right)},
\end{aligned}
$$

where $f$ is the frequency, $t$ is the time, $\tau$ is the positional parameter controlling the Gaussian window on the time axis $t$, and $\omega(f, \tau-t)$ is the Gaussian window function, and the height and width of which vary with the frequency $f$. At the same frequency, the Gaussian window function of different signals is fixed. In order to improve the energy concentration of time-frequency, parameters $\alpha$ and $\beta$ are introduced. The basic structure is as follows:

$$
\begin{aligned}
S(f, \tau) & =\int_{-\infty}^{+\infty} \frac{\left|f^{\alpha}\right|}{|\beta| \sqrt{2 \pi}} e^{\left(-f^{2 \alpha}(\tau-t)^{2} / 2 \beta^{2}\right)} x(t) e^{-j 2 \pi f t} \mathrm{~d} t \\
& =\int_{-\infty}^{+\infty} \omega(f,(\tau-t)) x(t) e^{-j 2 \pi f t} \mathrm{~d} t,
\end{aligned}
$$

where $\alpha$ and $\beta$ are adjustment factors and are generally positive. When $\beta$ increases or $\alpha$ decreases, the Gaussian 


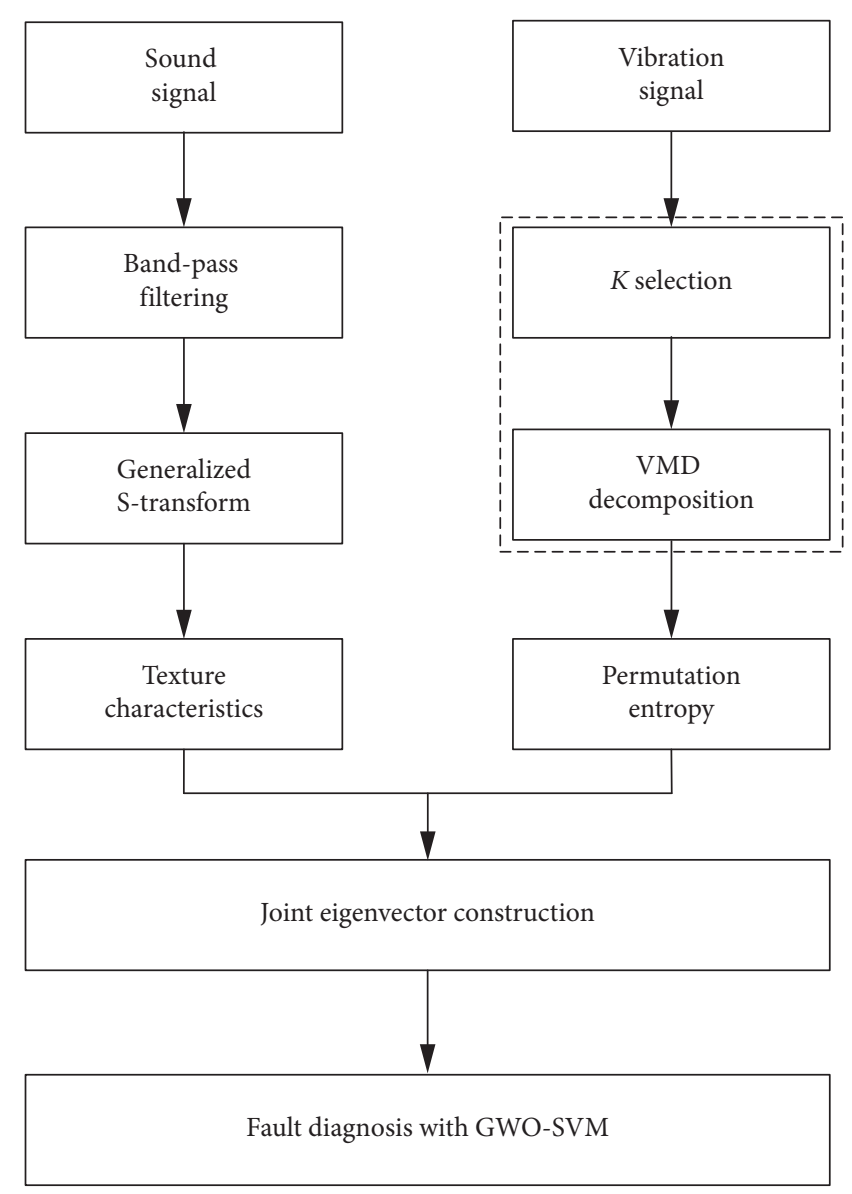

FIGURE 1: Diagnostic flowchart.
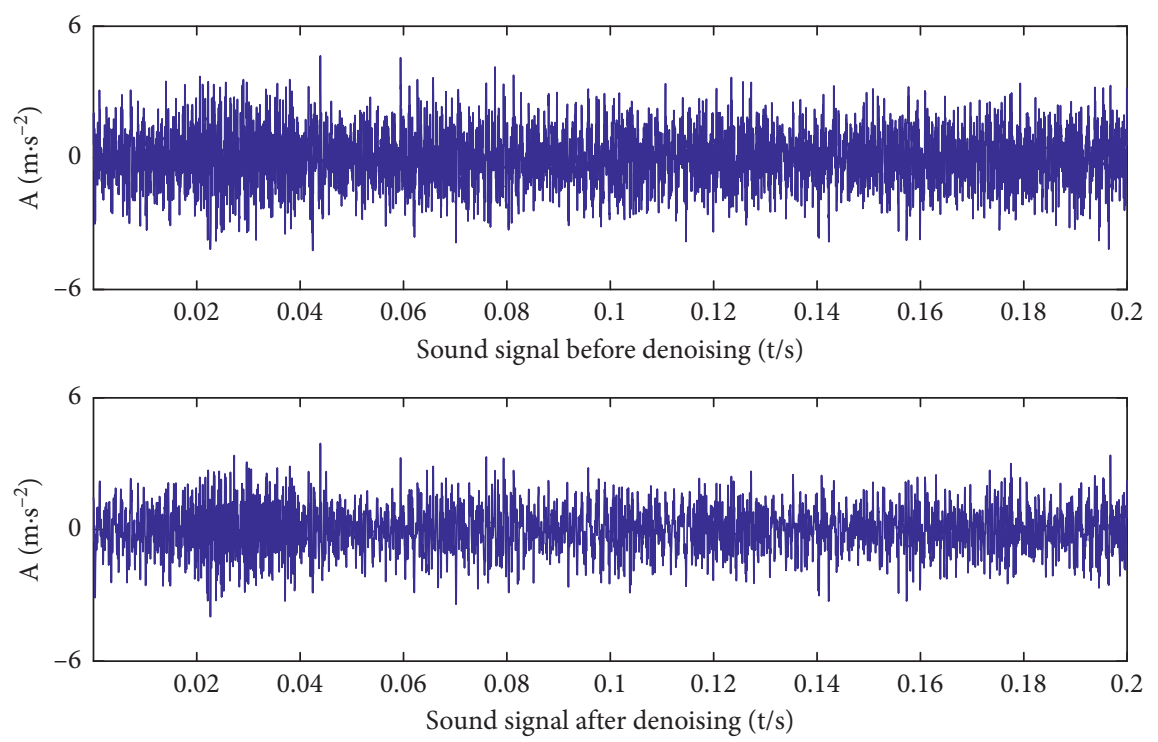

FIGURE 2: Comparison of results before and after denoising.

window width is stretched in the time domain and compressed in the frequency domain, and vice versa. The values of $\alpha$ and $\beta$ are selected as 1 and 2, respectively. The time-frequency diagram of the generalized S-transform of the vibration signal in the four states is shown in Figure 3.

The time-frequency texture images, after generalized Stransform, embody different texture features under different 


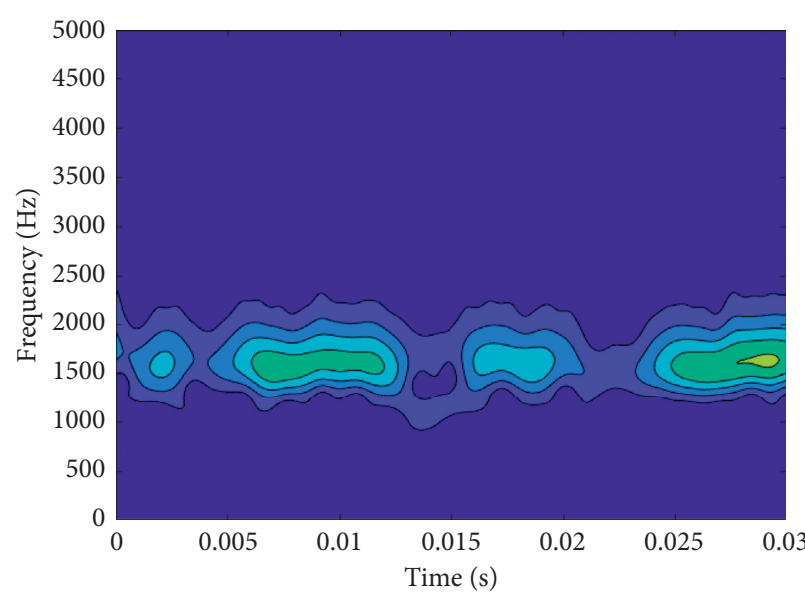

(a)

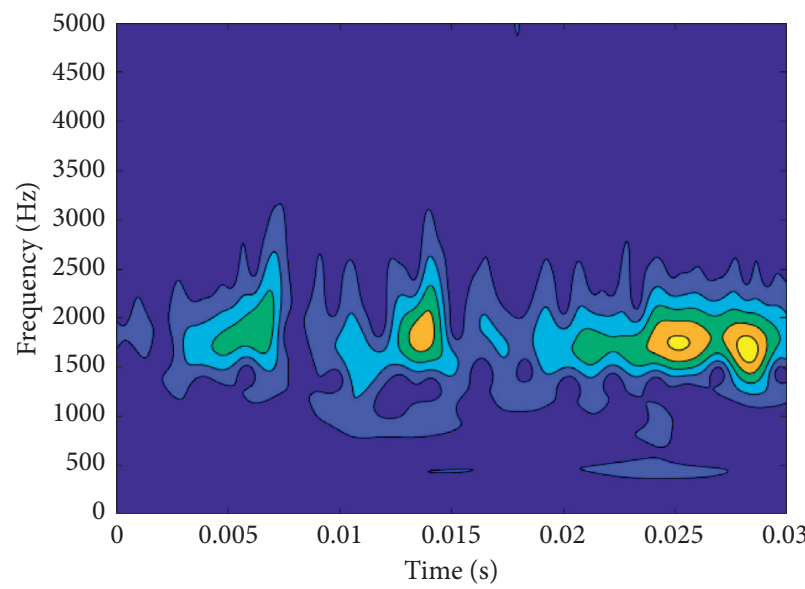

(c)

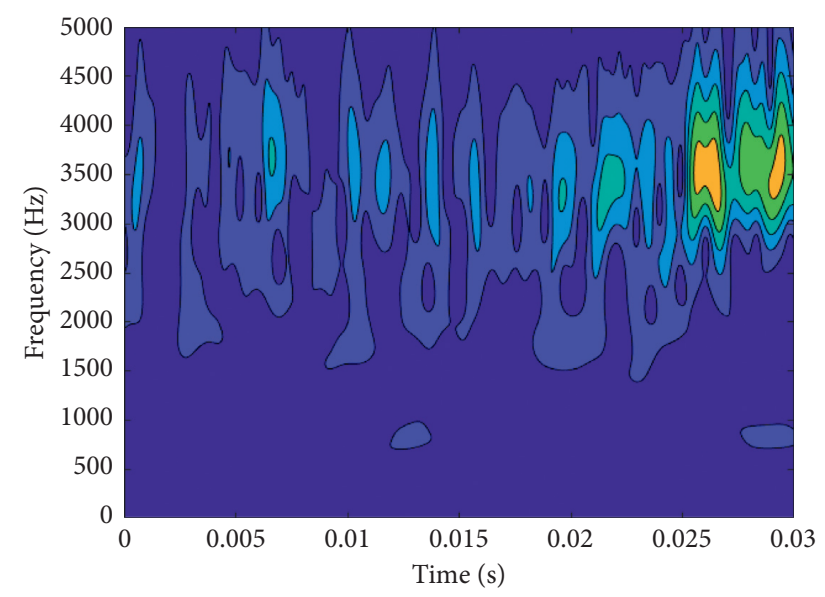

(b)

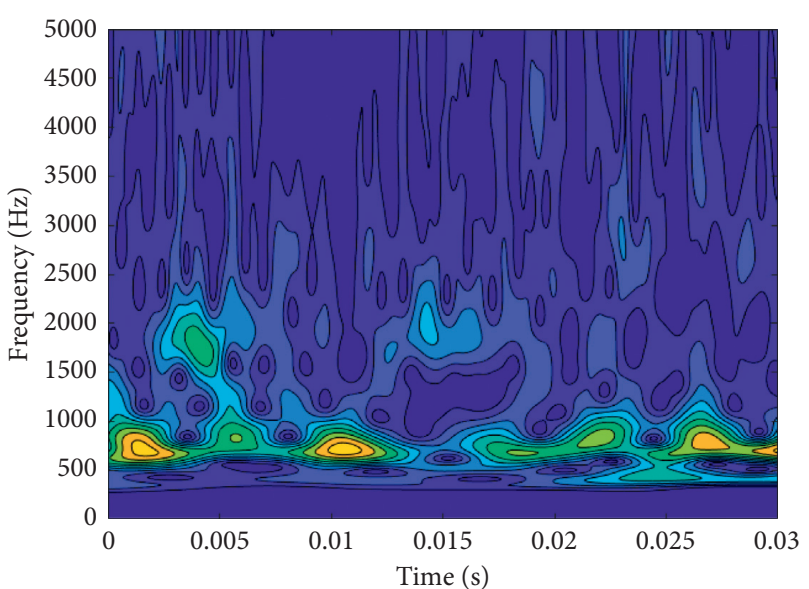

(d)

Figure 3: Time-frequency diagram of generalized S-transform in four states: (a) normal state, (b) belt damage, (c) retarder oil leakage, and (d) gear pitting peeling.

faults. The box dimension, directivity, and contrast ratio which reflect the time-frequency texture features are extracted as eigenvectors to classify faults.

2.2. Box Dimension. The box dimension is particularly sensitive to the texture roughness of the image spatial distribution and can quantitatively characterize the distribution law of the time-frequency diagram. The calculation process is as follows: subblocks of size $M \times M$ are divided into $L \times L$ $(1<L \leq M / 2, L$ is an integer), make $r=L / M$, then each grid is a column $L \times L \times L 1$ box, $L 1=L \times G / M$, and $G$ is gray level. Suppose the minimum and maximum values of the image grayscale in the $(i, j)$ grid fall in the $k$-th and $l$-th boxes, respectively, then the number of boxes required for the $(i, j)$ grid image is given by

$$
n_{r}(i, j)=k-l+1 .
$$

The number of boxes required to cover the entire image is $N_{r}$ :

$$
N_{r}=\sum n_{r}(i, j)
$$

The box dimension can be calculated by the following formula:

$$
D=\lim _{r \longrightarrow 0}\left[\frac{\log \left(N_{r}\right)}{\log (1 / r)}\right] .
$$

The $\log \left(N_{r}\right)$ and $\log (1 / r)$ slopes are fitted by leastsquares method, and the absolute value is the box dimension.

2.3. Directivity. Directivity describes the global characteristics of texture images and characterizes the trend of basic texture units and their arrangement tendency in all directions. Calculate the gradient vector $\Delta V$ of each pixel, whose modulus and direction are defined as follows:

$$
\left\{\begin{array}{l}
|\Delta V|=\frac{(|\Delta P|+|\Delta Q|)}{2}, \\
\theta=\tan ^{-1}\left(\frac{\Delta Q}{\Delta P}\right)+\frac{\pi}{2},
\end{array}\right.
$$


where $|\Delta V|$ is the gradient vector modulus; $\Delta P$ and $\Delta Q$ are the changes of $\Delta V$ in the horizontal and vertical directions, respectively; and $\theta$ is the angle of the gradient vector.

Make a histogram and calculate the directivity by calculating the peak value of the gradient statistical histogram. The formula is as follows:

$$
F_{\mathrm{dir}}=1-r \cdot n_{p} \times \sum_{p=1}^{n_{p}} \sum_{\varphi \in w_{p}}\left(\phi-\phi_{p}\right)^{2} \times H_{D}(\phi),
$$

where $w_{p}$ is the amplitude of the $p$-th peak, $n_{p}$ is the number of histogram peaks, the subscript $D$ of $H_{D}$ is the $D$-th peak of the histogram, and $r$ is the normalization factor.

2.4. Contrast Ratio. The contrast ratio reflects the difference of the image in gray level. The larger the difference in gray value, the stronger the contrast ratio. When there is a significant peak in the gray value of 0 or 255 , the deviation degree is measured by the kurtosis $k_{4}$, and the calculation is as follows:

$$
F_{c}=\frac{\sigma_{h}}{k_{4}},
$$

where $k_{4}=\mu_{4} / \sigma_{h}^{4}, \mu_{4}$ is the 4 th moment of the gray mean of the whole image and $\sigma_{h}$ is the standard deviation of the image.

Calculate the box dimension, directivity, and contrast ratio of the generalized S-transformed time-frequency diagram of the sound signal in the four states (belt breakage, retarder oil leakage, gear pitting peeling, and normal state), and record them as $D, F_{\mathrm{dir}}$, and $\mathrm{F}_{c}$, respectively. The sound texture features are shown in Table 1.

The texture features of the abovementioned sound signal have antinoise and rotation invariance and can describe the local pattern and arrangement rule of the image. Longitudinal and horizontal comparison of the characteristics of the sound texture can identify the operating state of the retarder.

\section{Vibration Signal Feature Extraction}

The energy spectrum of the vibration signal is concentrated within $10 \mathrm{kHz}$, and the vibration signals of the normal state and belt damage state of oil pumping machine are collected. The time domain waveform comparison is shown in Figure 4.

\subsection{VMD Decomposition}

3.1.1. VMD Decomposition Principle. The VMD decomposition is mainly divided into two parts: the establishment and solution of the variational constraint problem. The following problems are solved for the vibration signal with the data length $N$ in the oil production process:
TABLE 1: Sound texture features.

\begin{tabular}{lcccc}
\hline \multirow{2}{*}{ Operating state } & Group & \multicolumn{3}{c}{ Sound texture } \\
& & $\mathrm{D}$ & $F_{\text {dir }}$ & $\mathrm{F}_{c}$ \\
\hline \multirow{2}{*}{ Belt damage } & 1 & 2.5674 & 4.5897 & 1.2483 \\
\multirow{2}{*}{ Retarder oil leakage } & 2 & 2.5661 & 4.5812 & 1.2429 \\
\multirow{2}{*}{ Gear pitting peeling } & 1 & 3.8370 & 8.3845 & 2.2430 \\
\multirow{2}{*}{ Normal state } & 2 & 3.8367 & 8.3861 & 2.2423 \\
& 1 & 5.4279 & 6.3344 & 3.3394 \\
& 1 & 5.4286 & 6.3367 & 3.3323 \\
& 2 & 4.6391 & 5.4335 & 4.3428 \\
& & 4.6387 & 5.4345 & 4.3425 \\
\hline
\end{tabular}

$$
\begin{cases}\min _{\left\{u_{k}\right\},\left\{\omega_{k}\right\}} & \left\{\sum_{k}\left\|\partial_{t}\left[\left(\delta(t)+\frac{j}{\pi t}\right) * \mu_{k}(t)\right] e^{-j \omega_{k} t}\right\|^{2}\right\} \\ \text { s.t. } & \sum_{k} \mu_{k}=f,\end{cases}
$$

where $\left\{\mu_{p}\right\}:=\left\{\mu_{1}, \ldots, \mu_{p}\right\}$ is the decomposed $p$ modes and $\left\{\omega_{p}\right\}:=\left\{\omega_{1}, \ldots, \omega_{p}\right\}$ is the center frequency of the $p$ modes.

In order to solve the optimal solution, the quadratic penalty factor $\alpha$ and the Lagrangian operator $\lambda(t)$ are introduced to change the constrained variational problem into a nonbinding variational problem. The extended Lagrangian expression is as follows:

$$
\begin{aligned}
L\left(\left\{\mu_{k}\right\},\left\{\omega_{k}\right\}, \lambda\right)= & \alpha \sum_{k}\left\|\partial_{t}\left[\left(\delta(t)+\frac{j}{\pi t}\right) * \mu_{k}(t)\right] e^{-j \omega_{k} t}\right\|_{2}^{2} \\
& +\left\|f(t)-\sum_{k} \mu_{k}(t)\right\|_{2}^{2}+\langle\lambda(t), f(t) \\
& \left.-\sum_{k} \mu_{k}(t)\right\rangle .
\end{aligned}
$$

The saddle point of equation (13) is solved by the ADMM method, so that $\mu_{k}^{n+1}, \omega_{k}^{n+1}$, and $\lambda^{n+1}$ are continuously updated, and the modal component $\mu_{k}$ and center frequency $\omega_{k}$ are solved as follows:

$$
\begin{aligned}
\widehat{\mu}_{k}^{n+1}(\omega) & =\frac{\widehat{f}(\omega)-\sum_{i \neq k} \widehat{\mu}_{k}^{n}(\omega)+\hat{\lambda}(\omega) / 2}{1+2 \alpha\left(\omega-\omega_{k}\right)^{2}}, \\
\omega_{k}^{n+1} & =\frac{\int_{0}^{\infty} \omega\left|\hat{\mu}_{k}^{n}(\omega)\right| \mathrm{d} \omega}{\int_{0}^{\infty}\left|\widehat{\mu}_{k}^{n}(\omega)\right| \mathrm{d} \omega} .
\end{aligned}
$$

The VMD steps are as follows:

(1) Initialize $\hat{\mu}_{k}^{1}, \omega_{k}^{1}, \lambda^{\hat{1}}$, and $n$, let its initial value be 0 , and set the decomposition modal number $K$ to 2 (predecomposition optimization).

(2) Update $\mu_{k}$ and $w_{k}$ according to equations (14) and (15), respectively. 

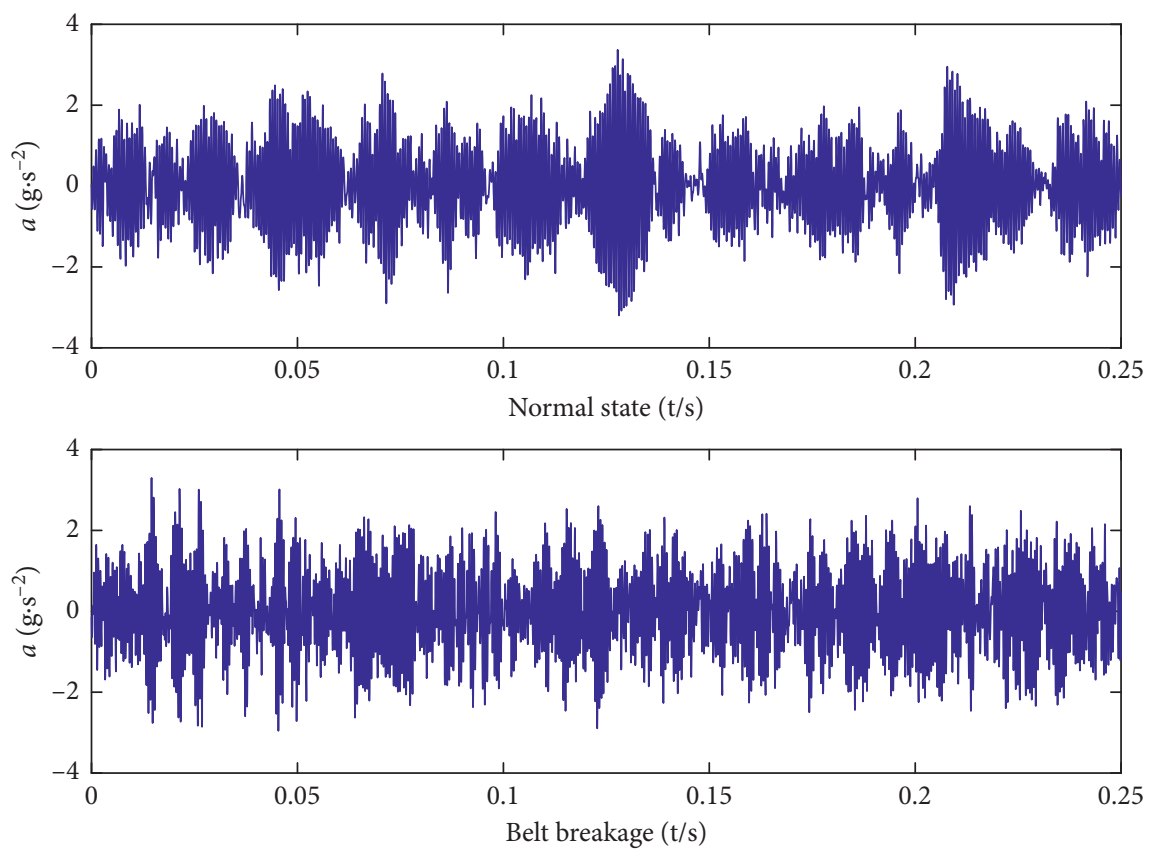

FIgURE 4: Comparison of normal and abnormal waveforms in time domain.

(3) Update $\hat{\lambda}$ :

$$
\hat{\lambda}^{n}(\omega)+\tau\left[\hat{f}(\omega)-\sum \widehat{\mu}_{k}^{n+1}\right] \longrightarrow \hat{\lambda}^{n+1}(\omega) .
$$

(4) If the following formula is satisfied, the iteration is stopped and the result is output; otherwise, it returns to step 2:

$$
\frac{\sum\left\|\widehat{\mu}_{k}^{n+1}-\widehat{\mu}_{k}^{n}\right\|_{2}^{2}}{\left\|\widehat{\mu}_{k}^{n}\right\|_{2}^{2}<\varepsilon} .
$$

3.1.2. VMD Parameter K Optimization. In order to prevent the VMD from being decomposed, the $K$ parameter is selected according to the energy conservation theory before and after decomposition. For the original retarder vibration signal sequence $x(i)$, the energy calculation formula is as follows:

$$
E=\sqrt{\frac{\sum_{i=1}^{n} x^{2}(i)}{n}},
$$

where $E$ represents the signal energy value and $n$ is the sampling point. In order to characterize the energy difference before and after the VMD decomposition, the energy difference parameter $\psi$ is defined and calculated as follows:

$$
\psi=\frac{\left|\sum_{x=1}^{K} E_{x}-E\right|}{E} \times 100 \% \text {, }
$$

where $E_{x}$ corresponds to the energy of the $x$ component, $K$ is the number of components, and $E$ is the original signal energy. The total energy of $K$ components after VMD decomposition is equal to the energy of the original signal (i.e., the ideal value of $\psi$ is 0 ), after many experiments and calculations, the trend of $K$ is shown in Figure 5.
It can be seen from Figure 5 that when $K$ is greater than 6 , the energy difference parameter $\lambda$ is increased, and it can be judged that overdecomposition occurs. At this time, the $K$ value at the turning point is the optimal decomposition mode number of the VMD. The time-frequency diagram obtained by decomposing the vibration signal in the belt damage state is shown in Figures 6 and 7.

It can be seen from the frequency domain spectrum of Figure 7 that the VMD decomposition of the vibration signal effectively improves the modal aliasing phenomenon and provides a strong support for accurate fault diagnosis.

3.2. Calculated Permutation Entropy. Permutation entropy can detect the sudden change of the signal, has strong antinoise ability and high time resolution, and is highly targeted to the nonstationary chaotic vibration signal under complex environment of oil production field.

For the signal sequence $\{X(i), i=1,2, \ldots, n\}$, phase space reconstruction is performed to obtain the following matrix:

$$
\left[\begin{array}{cc}
x(1) & x(1+\tau) \cdots x(1+(m-1) \tau) \\
x(2) & x(2+\tau) \cdots x(2+(m-1) \tau) \\
x(j) & x(j+\tau) \cdots x(j+(m-1) \tau) \\
\cdots & \cdots \\
x(k) & x(k+\tau) \cdots x(k+(m-1) \tau)
\end{array}\right]
$$

where $j=1,2, \ldots, k, k=n-(m-1) \tau$, and $m$ and $\tau$ are the embedded dimension and delay time. The elements of each component (total of $k$ ) are arranged in ascending order according to the numerical value, and different symbol sequences are obtained according to the reconstructed component index. The $m$-dimensional phase space mapping 


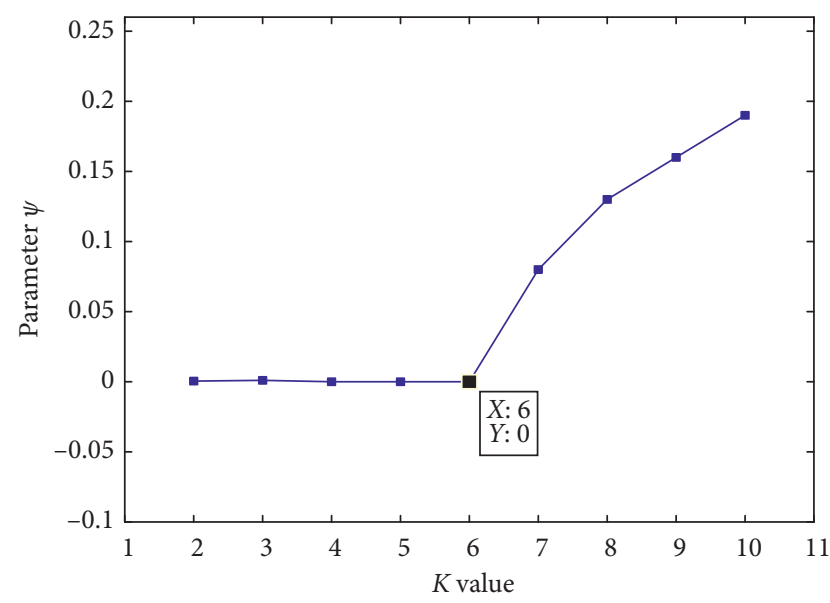

Figure 5: Change trend of $K$ value.

symbol sequence has $m$ ! species, and the probability of occurrence of $s$ different symbol sequences is $P_{1}, P_{2}, \ldots, P_{s}$, respectively, calculated as follows:

$$
P e(m)=-\sum_{j=1}^{s} P_{j} \ln P_{j} .
$$

Normalize $P e(m)$ :

$$
0 \leq P e=\frac{P e(m)}{\ln (m !) \leq 1} .
$$

After many experiments and comparative analysis, the embedding dimension $m$ is selected as 8 and the delay time $\tau$ is selected as 5, and the entropy of the six modes of the vibration signal is obtained as shown in Table 2 .

The entropy value is related to the degree of the sample sequence rule. From Table 2, it can be seen that there are obvious differences in the permutation entropy of each state, the distinguishability is good, and the state information of the retarder can be effectively characterized.

\section{Experiment and Result Analysis}

4.1. GWO-SVM Diagnostic Model. SVM is especially suitable for small sample fault diagnosis, but the selection of penalty factor $C$ and kernel function directly affect the classification performance of SVM. Considering that the sample in this paper is a multisensor data feature set, the sensor frequency response, environmental noise, and load output fluctuation interference have a great influence on the diagnostic accuracy, and GA, PSO, and other algorithms are easy to fall into the local optimal solution, so a mixed kernel function is constructed and the gray wolf optimization (GWO) algorithm is introduced to find the best penalty factor and mixing coefficient.

It is necessary to combine the different kernel functions to obtain a kernel function with strong promotion ability and learning ability and good extrapolation ability. The Taylor-Kernel with moderate decreasing (T-KMOD) function satisfies the zero-point near-descent criterion, with good flexibility, fast convergence, and good locality.
Polynomial functions have high classification accuracy and strong generalization ability. Therefore, the two are combined in the following form:

$$
S_{M}=\lambda S_{\text {poly }}+(1-\lambda) S_{\mathrm{T}-\mathrm{KMOF}},
$$

where $S_{\mathrm{T}-\mathrm{KMOD}}=\mathrm{T}-\operatorname{KMOD}\left(x, x^{\prime}\right)=L \sum_{i=1}^{n}\left(\gamma /\left(\left\|x-x^{\prime}\right\|^{2}+\right.\right.$ $\left.\left.\sigma^{2}\right)\right)^{k * i}, S_{\text {poly }}=\left[\left(x \cdot x^{\prime}\right)+C\right]^{q}, \lambda \in(0,1) C \geq 0 ; x \cdot x^{\prime} \in R^{n}$, $L>0, L$ is used to control the value of the kernel function at 0 . $\sigma$ and $\gamma$ are used to control the width and convergence rate of the kernel function, respectively, and both $i$ and $n$ are positive integers.

GWO has a fast convergence speed and a simple structure, and it is easier to achieve optimal classification. The mathematical model is as follows:

$$
\left\{\begin{array}{l}
B=\left|C \cdot X_{p}(t)-X(t)\right| \\
X(t+1)=X_{p}(t)-A \cdot B,
\end{array}\right.
$$

where $t$ represents the current number of iterations; $X_{p}(t)$ is the prey position vector, and $A$ and $C$ are coefficient vectors. $A$ and $C$ are as follows:

$$
\left\{\begin{array}{l}
A=2 a \cdot r_{1}-a, \\
C=2 r_{2},
\end{array}\right.
$$

where $a$ is the convergence factor, satisfying $a \in[0,2]$, and $r_{1}$ and $r_{2}$ are random vectors in $[0,1]$.

60 sets of samples were collected for each state, 40 sets were used for training, and 20 sets were used for testing. Initialize the population, calculate the fitness value of each wolf, select the first three optimal fitness values, determine the gray wolf rank, and update the gray wolf position, head wolf, coefficient vector, and other parameters until the SVM parameters are optimal. The algorithm flowchart is shown in Figure 8:

4.2. Experimental Result. In the experiment, the MVP-6000 acquisition card of ADLINK Company is used, whose sampling rate was set to $40 \mathrm{kHz}$. The sound sensor is placed about $50 \mathrm{~cm}$ away from the sound source, and the vibration sensor is adsorbed on the surface of the vibration body of the retarder. The acquisition card is equipped with IEPE constant current source, $\pm 10 \mathrm{~V}$ voltage range, 24 bit resolution, and $110 \mathrm{~dB}$ dynamic range.

The sound texture feature extracted by the generalized Stransform and the vibration entropy feature calculated by the VMD decomposition are combined to construct a joint eigenvector matrix and sent to the SVM for training. GWO is used to optimize the penalty factor $C$ and the mixing coefficient $\lambda$ in order to improve the SVM classification performance. The number of iterations is set to 100, and the optimal parameter values are 2.5693 and 0.17 , respectively. The convergence of the algorithm is shown in Figure 9:

The sound-vibration joint eigenvectors are constructed with a combination of Tables 1 and 2 . Set the normal state sample characteristic value label to 1 (1-20 groups), the belt damage is 2 (21-40 groups), the retarder oil leakage is 3 

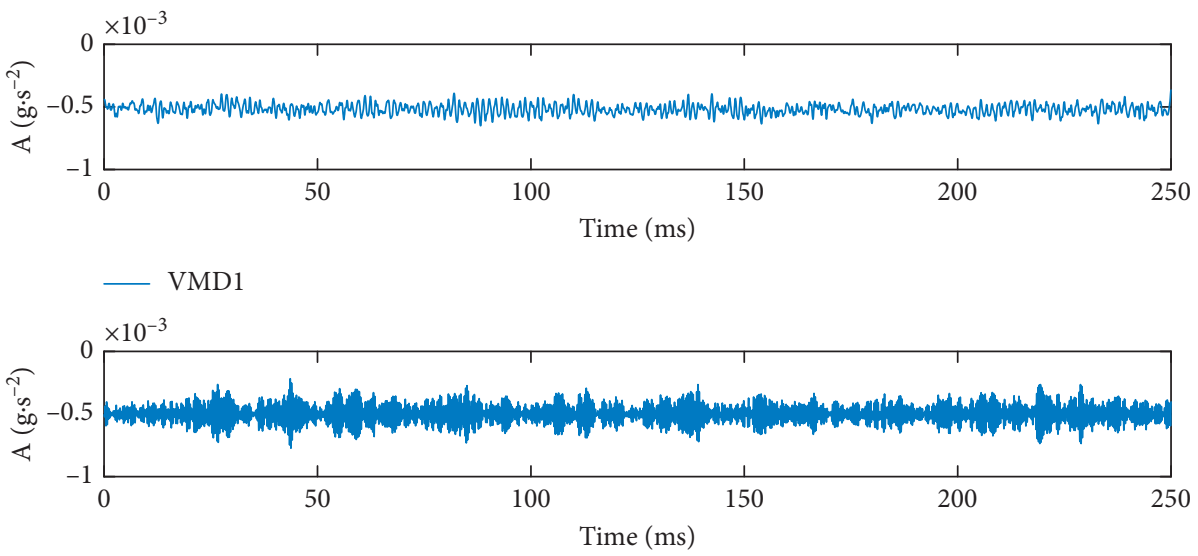

- VMD2

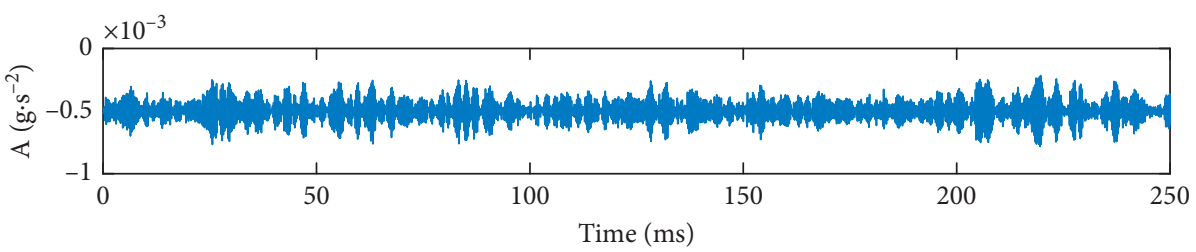

- VMD3

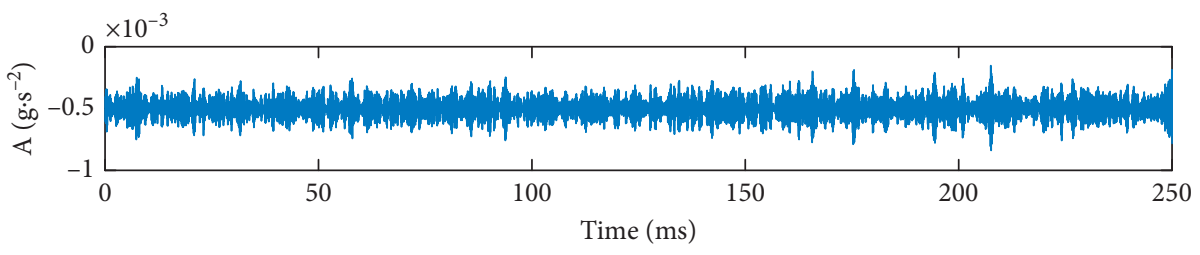

- VMD4

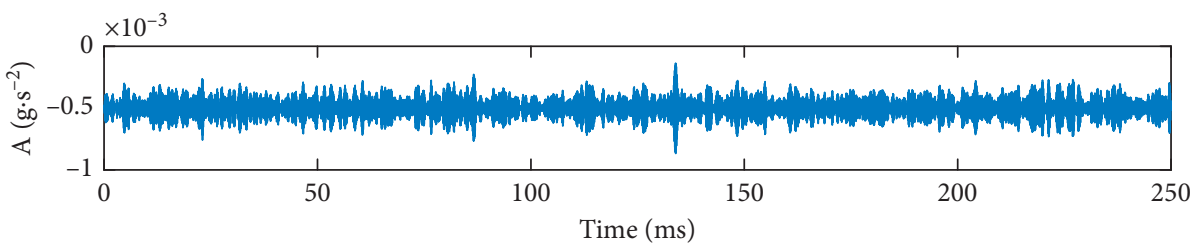

- VMD5

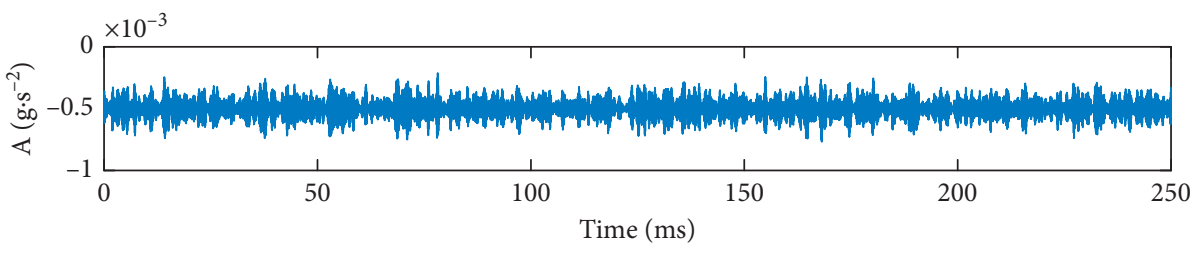

VMD6

Figure 6: Time domain diagram of vibration signal VMD decomposition.

(41-60 groups), and the gear pitting peeling is 4 (61-80 groups). Some test data are shown in Table 3.

The diagnosis results are as follows, in which Figures 10 and 11 show the fault identification results before and after the model optimization.

According to the results of Figure 10, two groups of normal state samples were misjudged as belt damage state, two groups of retarder oil leakage state samples were misjudged as belt damage state, and one group of gear pitting peeling state samples was misjudged as retarder oil leakage state. The identification accuracy is $93.75 \%$. The results of the GWO-SVM model are shown in Figure 11. All 80 groups of test samples are classified correctly, and the identification accuracy is $100 \%$. Although the input characteristics are the same, the diagnostic accuracy differs significantly. Compared with SVM, the accuracy of GWO-SVM is improved by $6.25 \%$. The reason is that GWO-SVM diagnostic model can find the appropriate parameters of SVM classifier through GWO, which makes full use of the classification advantages of SVM in constructing optimal hyperplane, and thus, the 

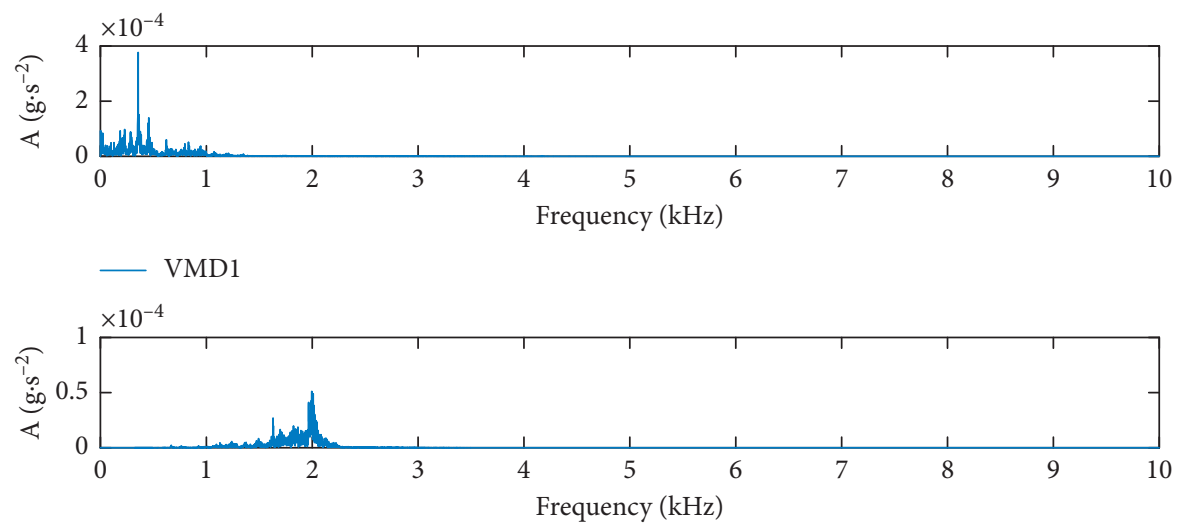

- VMD2

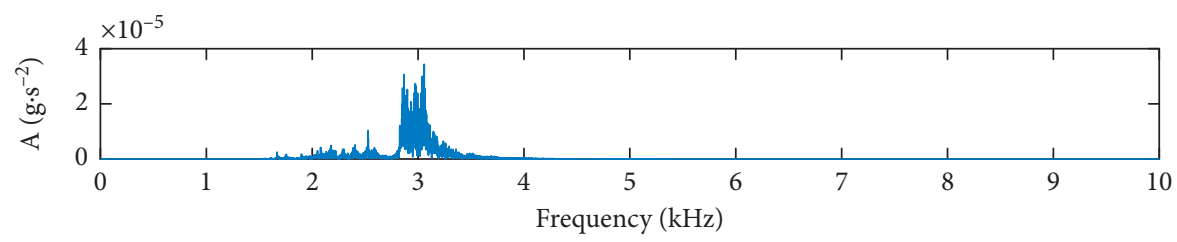

- VMD3

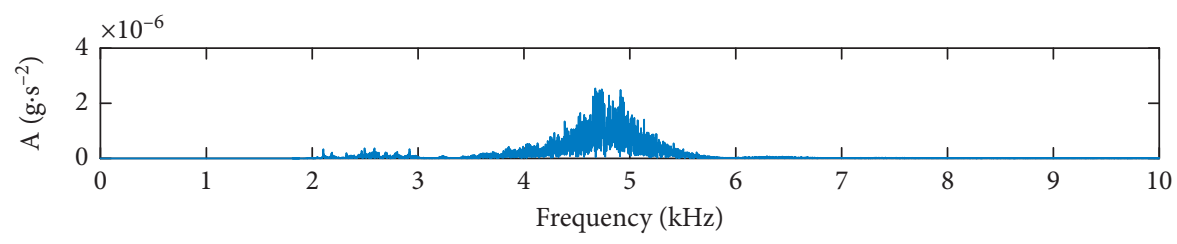

- VMD4

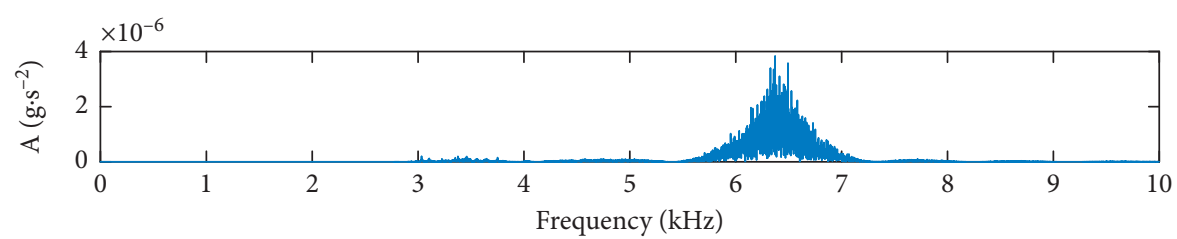

- VMD5

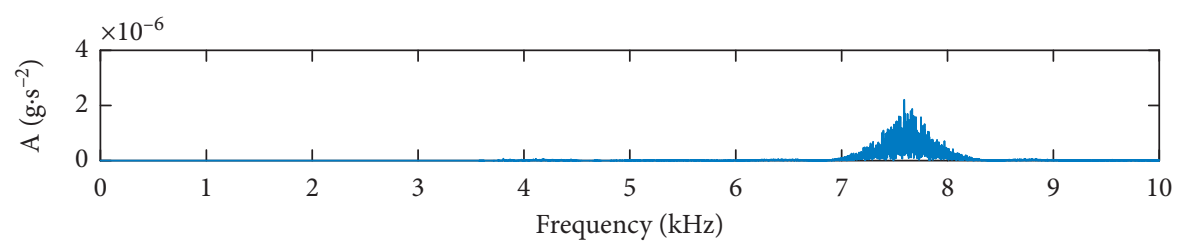

— VMD6

FIGURE 7: Frequency domain diagram of vibration signal VMD decomposition.

TABle 2: Permutation entropy of modal components of vibration signals.

\begin{tabular}{|c|c|c|c|c|c|c|c|}
\hline \multirow{2}{*}{ Operating state } & \multirow{2}{*}{ Group } & \multicolumn{6}{|c|}{ Vibration entropy } \\
\hline & & $V_{1}$ & $V_{2}$ & $V_{3}$ & $V_{4}$ & $V_{5}$ & $V_{6}$ \\
\hline \multirow{2}{*}{ Belt damage } & 1 & 0.6139 & 0.6753 & 0.6214 & 0.6457 & 0.6390 & 0.6523 \\
\hline & 2 & 0.6138 & 0.6751 & 0.6211 & 0.6457 & 0.6391 & 0.6522 \\
\hline \multirow{2}{*}{ Retarder oil leakage } & 1 & 0.3269 & 0.3658 & 0.3569 & 0.3546 & 0.3426 & 0.3261 \\
\hline & 2 & 0.3265 & 0.3659 & 0.3565 & 0.3546 & 0.3425 & 0.3260 \\
\hline \multirow{2}{*}{ Gear pitting peeling } & 1 & 0.5689 & 0.5469 & 0.5218 & 0.5634 & 0.5849 & 0.5459 \\
\hline & 2 & 0.5687 & 0.5468 & 0.5217 & 0.5634 & 0.5847 & 0.5458 \\
\hline \multirow{2}{*}{ Normal state } & 1 & 0.1232 & 0.1526 & 0.1726 & 0.1521 & 0.1358 & 0.1189 \\
\hline & 2 & 0.1231 & 0.1523 & 0.1725 & 0.1523 & 0.1356 & 0.1189 \\
\hline
\end{tabular}




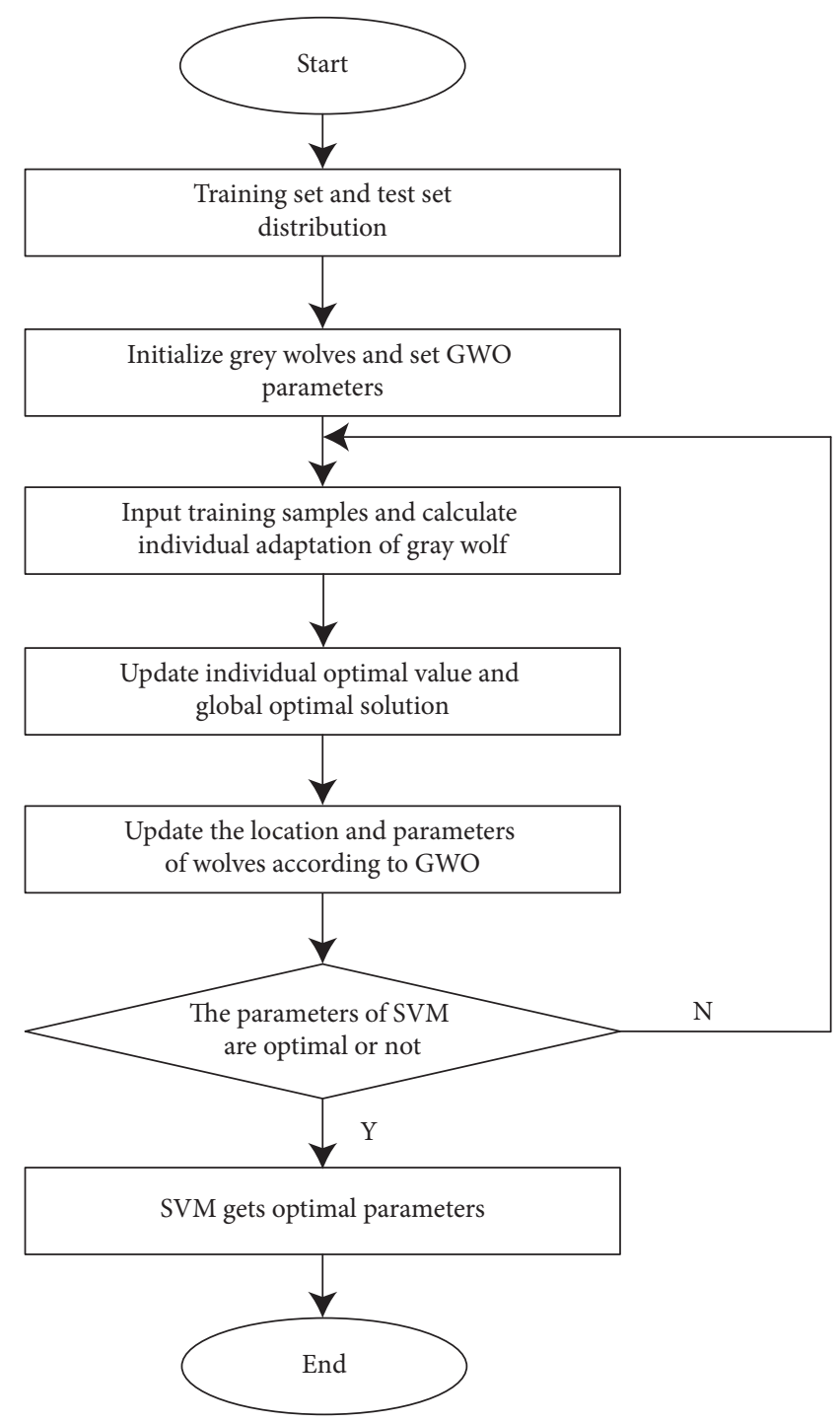

FIgURE 8: Gray wolf optimization algorithm flowchart.

accuracy of fault diagnosis is improved. At the same time, the important influence of the accurate selection of penalty factor $C$ and kernel function on the accuracy of state identification is verified. Compared with the SVM model, GWO-SVM can more accurately reflect the type of retarder defects.

\subsection{Diagnostic Effect Comparison and Verification}

4.3.1. Sound-Vibration Joint Characteristic Method Verification. Contrast the diagnosis effect of sound signal, vibration signal, sound-vibration combined, as shown in Figure 12. For the experimental sample data, the diagnostic accuracy of sound characteristics and vibration characteristics were $91 \%$ and $94 \%$, respectively, and the sound-vibration combined diagnosis accuracy rate reached $100 \%$. Therefore, based on the combined characteristics of sound

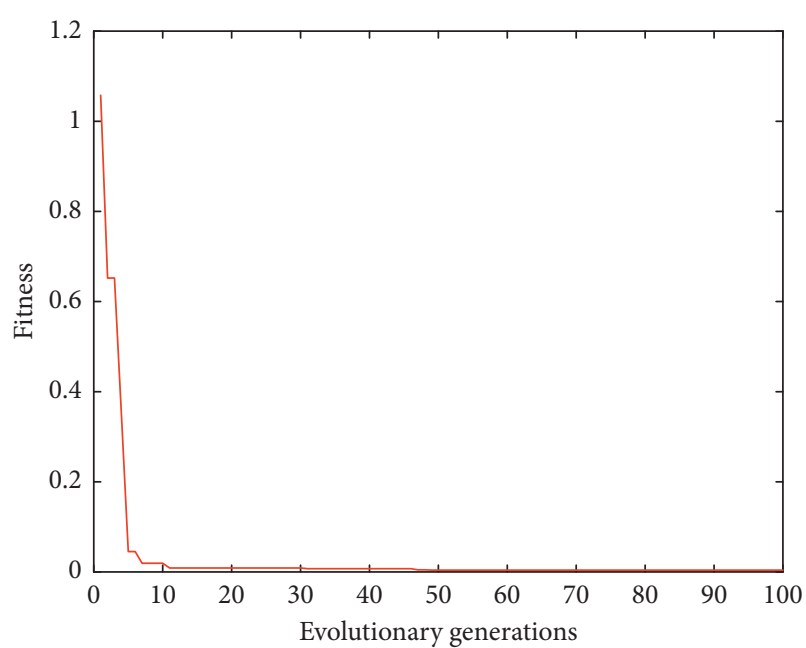

Figure 9: Convergence of GWO algorithm. 
TABle 3: Partial test samples and diagnostic results.

\begin{tabular}{|c|c|c|c|c|c|c|c|c|c|c|}
\hline \multirow[b]{2}{*}{$D$} & \multirow[b]{2}{*}{$F_{\text {dir }}$} & \multirow[b]{2}{*}{$\mathrm{F}_{c}$} & \multicolumn{3}{|c|}{ Joint eigenvector } & \multirow[b]{2}{*}{$\mathrm{V}_{4}$} & \multirow[b]{2}{*}{$\mathrm{V}_{5}$} & \multirow[b]{2}{*}{$\mathrm{V}_{6}$} & \multirow{2}{*}{ Diagnosis label } & \multirow{2}{*}{ Diagnosis result } \\
\hline & & & $\mathrm{V}_{1}$ & $\mathrm{~V}_{2}$ & $\mathrm{~V}_{3}$ & & & & & \\
\hline 4.6393 & 5.4331 & 4.3423 & 0.1233 & 0.1525 & 0.1728 & 0.1523 & 0.1360 & 0.1190 & 1 & \multirow{2}{*}{ Normal state } \\
\hline 4.6385 & 5.4348 & 4.3422 & 0.1229 & 0.1523 & 0.1723 & 0.1523 & 0.1358 & 0.1193 & 1 & \\
\hline 2.5673 & 4.5897 & 1.2485 & 0.6137 & 0.6752 & 0.6213 & 0.6459 & 0.6391 & 0.6527 & 2 & \multirow{2}{*}{ Belt damage } \\
\hline 2.5665 & 4.5813 & 1.2430 & 0.6139 & 0.6753 & 0.6209 & 0.6458 & 0.6392 & 0.6523 & 2 & \\
\hline 3.8371 & 8.3847 & 2.2431 & 0.3271 & 0.3661 & 0.3570 & 0.3541 & 0.3428 & 0.3265 & 3 & \multirow{2}{*}{ Retarder oil leakage } \\
\hline 3.8365 & 8.3859 & 2.2425 & 0.3267 & 0.3660 & 0.3566 & 0.3542 & 0.3427 & 0.3261 & 3 & \\
\hline 5.4276 & 6.3346 & 3.3395 & 0.5692 & 0.5468 & 0.5219 & 0.5633 & 0.5851 & 0.5463 & 4 & \multirow{2}{*}{ Gear pitting peeling } \\
\hline 5.4282 & 6.3361 & 3.3325 & 0.5685 & 0.5471 & 0.5219 & 0.5639 & 0.5849 & 0.5465 & 4 & \\
\hline
\end{tabular}

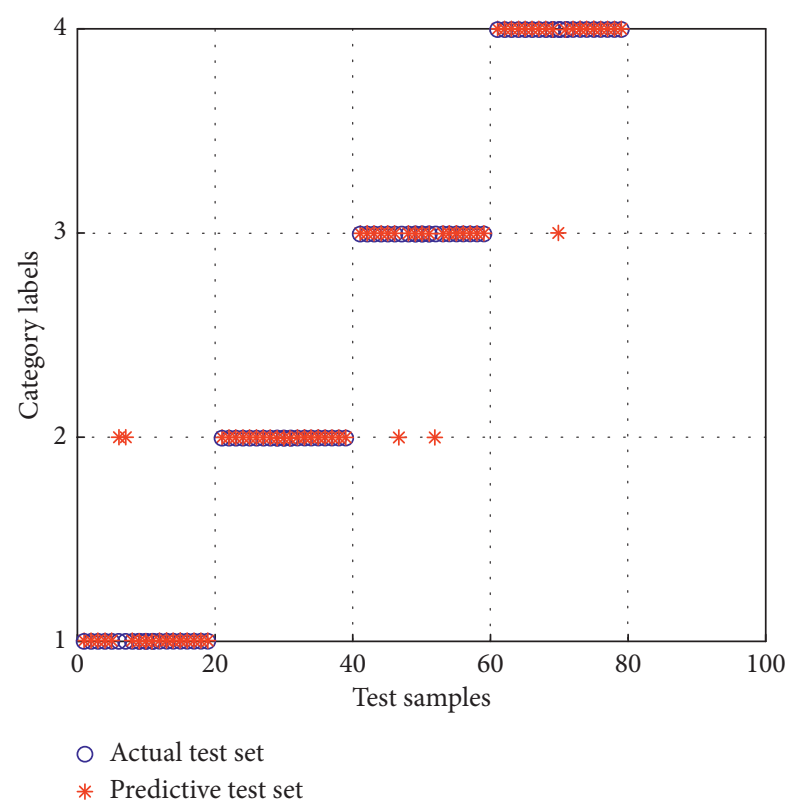

FIgURE 10: Fault identification results of the original SVM model.

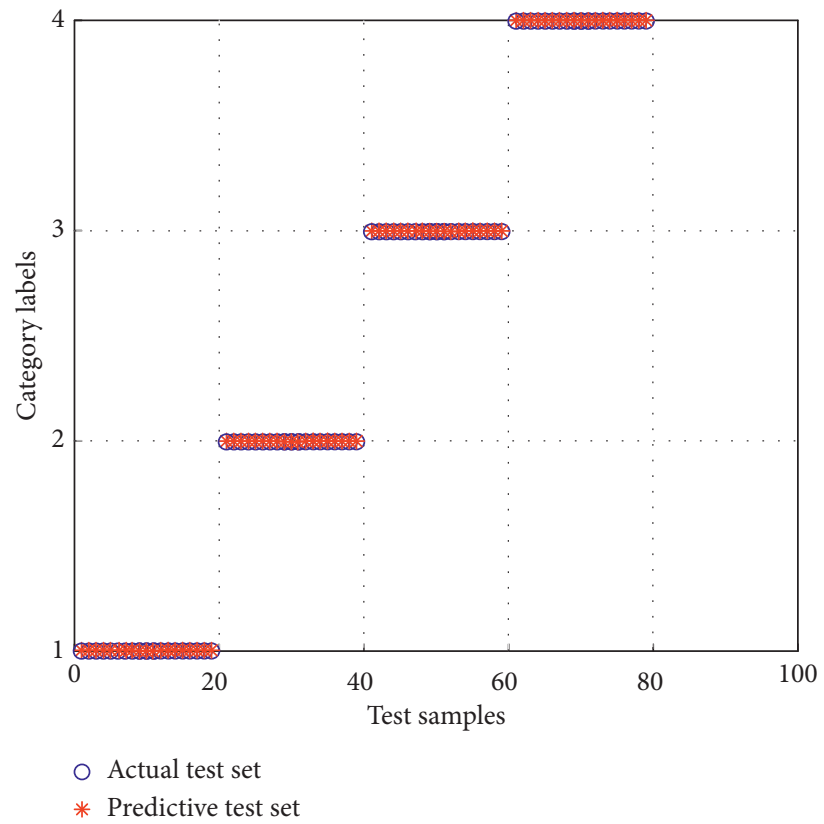

FIgURE 11: Fault identification results of the GWO-SVM model. 


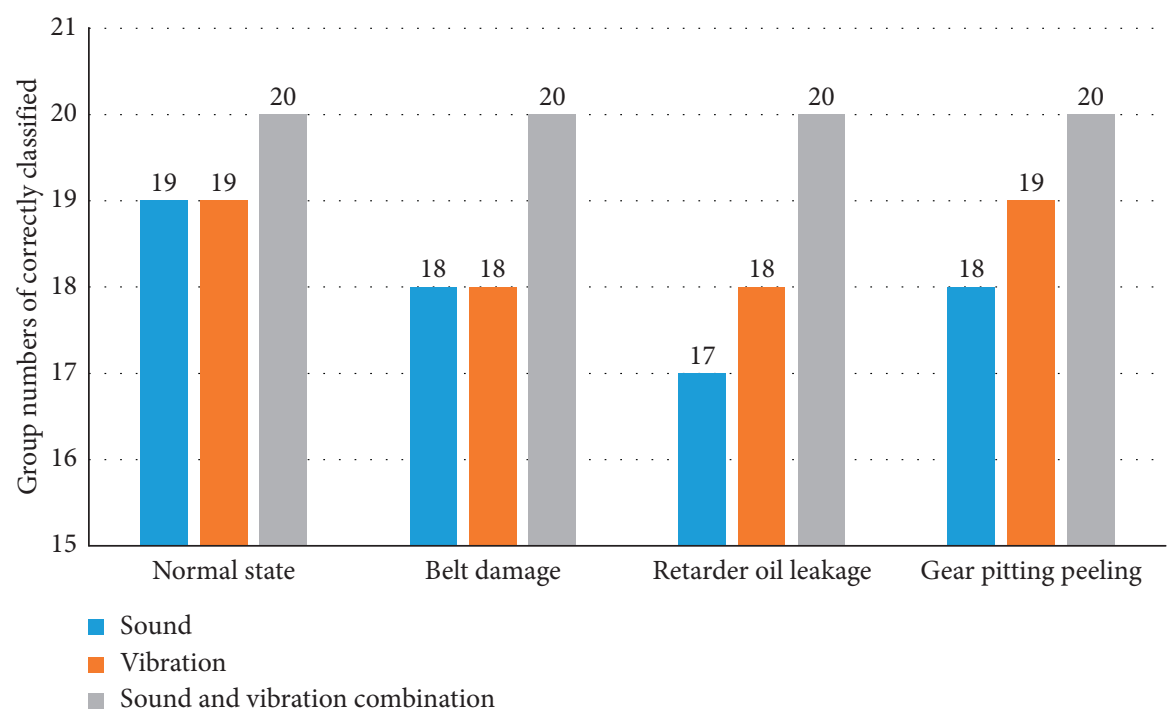

FiguRE 12: Comparison of three eigenvectors.

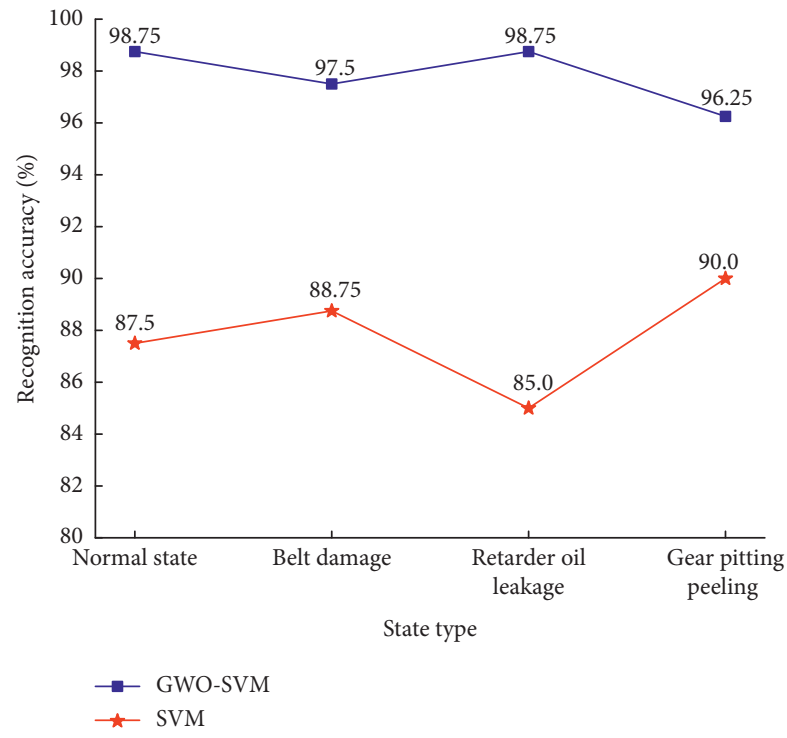

Figure 13: Accuracy comparison in four states.

and vibration, the state information of the retarder can be fully reflected, the extracted eigenvectors are complementary, and the diagnostic effect is improved.

\subsubsection{Verification of Generalization Performance.} Because the source and structure of the data are different in the actual oilfield operation, it is necessary to classify the fault data of the same type and different characteristics. In the generalization experiment, the sampling rate was changed from $40 \mathrm{kHz}$ to $30 \mathrm{kHz}$, and the PCB357B21 type vibration sensor and the WM-025N type pickup were replaced, and the sensor placement position was changed. The diagnosis result is shown in Figure 13.

It can be seen from Figure 13 that the overall diagnostic accuracy of the optimized SVM model still reaches $97.8 \%$ in the case of changes in acquisition parameter settings, sensor types, and positions, which is much higher than the unoptimized model, indicating that the optimized model has stronger adaptability to fresh samples and better generalization ability.

\section{Conclusion}

Defect identification of related retarder of beam oil pumping machine has always been a technical problem in the state monitoring of distributed oil production wells. The combination of sound texture and vibration entropy characteristics and the GWO-SVM classification algorithm, proposed in this paper, can effectively and accurately diagnose the field faults under the complementary frequency band. The main contributions and novels of the proposed method are summarized as follows:

(1) A fault diagnosis method based on the complementarity combination of sound-vibration signals is proposed for retarder equipment of oil pumping machine, which improves the accuracy of fault identification on the basis of nonmissing detection of retarder defects.

(2) For sound signals, the box dimension, directivity, and contrast ratio of time-frequency diagram are calculated after generalized S-transform to construct the sound texture features. For vibration signals, parameter $K$ of the VMD method is selected by the energy method, and the permutation entropy of modal components is obtained to construct the vibration entropy characteristics. The combination of the two effectively characterizes comprehensive information on various types of fault samples.

(3) The diagnosis model of GWO-SVM proposed in this paper, which optimizes the penalty factor and mixing coefficient, greatly improves the accuracy and generalization of SVM identification. It has broad 
application value in fault diagnosis of oil well machinery and equipment.

\section{Data Availability}

The data used to support the findings of this study are available from the corresponding author upon request.

\section{Conflicts of Interest}

The authors declare that they have no conflicts of interest.

\section{Authors' Contributions}

All the authors have made a contribution to a great extent: Shutao Zhao provided innovation and ideas of the paper; Erxu Wang and Ke Chang wrote the article and revised it later; Bo Li translated the content of the article; Kedeng Wang and Qingquan Wu collected the experimental data.

\section{Acknowledgments}

This research was supported by State Key Laboratory of New Energy and Power Systems, and obtained experimental data from Scientific and Technological Projects (Project no: HBYT-ZBZX-FWZB-2018-0716).

\section{References}

[1] J. P. Wang, Fault Diagnosis Technology and Application of Machinery and Equipment, Northwest Polytechnic University Press, Xi'an, China, 2001.

[2] Z. Tian, M. J. Zuo, and S. Wu, "Crack propagation assessment for spur gears using model-based analysis and simulation," Journal of Intelligent Manufacturing, vol. 23, no. 2, pp. 39-53, 2009.

[3] J. Wang, The Gearbox Fault Diagnosis System of Pumping Gearbox, Northeast Petroleum University, Daqing, China, 2015.

[4] X. Zeng, Research on Transmission Fault Classification and Recognition Based on Convolution Neural Network, South China University of Technology, Guangzhou, China, 2016.

[5] M. Landry, F. Leonard, C. Landry, R. Beauchemin, O. Turcotte, and F. Brikci, "An improved vibration analysis algorithm as a diagnostic tool for detecting mechanical anomalies on power circuit breakers," IEEE Transactions on Power Delivery, vol. 23, no. 4, pp. 1986-1994, 2008.

[6] Q. LI, Fault Diagnosis of Gearbox Based on Wavelet Transform and Support Vector Machine, Jilin University, Changchun, China, 2017.

[7] Z. Jiang, J. Ding, H. Li et al., "Research on tooth surface damage detection of automobile main reducer based on EMD and hilbert analysis," Mechanical Transmission, vol. 37, no. 12, pp. 9-13, 2013.

[8] W. Sun, B. Xiong, J. Huang et al., "Fault diagnosis method for rolling bearings based on wavelet packet noise reduction and LMD," Journal of Vibration \& Shock, vol. 31, no. 18, pp. 153-156, 2012.

[9] Z. Xiaoxia, Z. Guowang, R. Haohan et al., "A rolling bearing fault diagnosis method based on variational mode decomposition and permutation entropy," Journal of Vibration and Shock, vol. 36, no. 22, pp. 22-28, 2017.
[10] Z. Ma, Y. Li, L. Zheng et al., "Fault feature extraction of rolling bearing based on variational mode decomposition and teager energy operator," Journal of Vibration and Shock, vol. 35, no. 13, pp. 134-139, 2016.

[11] S. Zhang, Y. Cai, L. Shi et al., "Fault diagnosis of internal combustion engine based on discrete generalized S transform and bidirectional two-dimensional principal component analysis," China Mechanical Engineering, vol. 29, no. 8, pp. 899-905, 2018.

[12] S. Zhao, P. Zhang, Shenlu et al., "Vibration and acoustic joint mechanical fault diagnosis method of high voltage circuit breakers," Journal of Electrical Technology, vol. 29, no. 7, pp. 216-221, 2014.

[13] S. Zhao, Y. Wang, M. Li et al., "Circuit breaker fault diagnosis method based on sound-vibration joint characteristic entropy," Journal of North China Electric Power University, vol. 43, no. 6, pp. 20-24, 2016.

[14] P. Zhang and X. Zhou, "Mechanical fault diagnosis of high voltage circuit breaker based on three-dimensional vibrationacoustics-time diagram," High-voltage Electrical Appliances, vol. 50, no. 12, pp. 121-125, 2014.

[15] H. Mengyuan, D. Qiaolin, Z. Shutao et al., "Research of circuit breaker intelligent fault diagnosis method based on double clustering," IEICE Electronics Express, vol. 14, no. 17, Article ID 20170463, 2017.

[16] Y. Guo, Y. Wei, X. Zhou et al., "S-transform is used to extract shock characteristics of rolling bearing fault signals," Vibration Testing and Diagnosis, vol. 34, no. 5, pp. 818-822, 2014.

[17] J. Wang and W. Wang, "Fault diagnosis of pumping unit reducer based on RBF network," Petroleum Machinery, vol. 35 , no. 1, pp. 28-31, 2007. 